\title{
Experiencia académica escolar: siembra y seguimiento de plantas nativas para la recuperación ambiental del Macizo de Iguaque, Villa de Leyva, Colombia
}

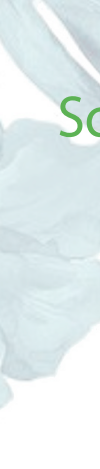

School academic experience: planting and monitoring native plants for the environmental recovery of the Iguaque Massif, Villa de Leyva, Colombia

\author{
Ivonne Lorena Reina Fonseca* \\ Andrés Felipe Cuadrado Hernández ${ }^{* *}$ \\ Juan Carlos Salas Castañeda*** \\ Javier Ernesto Cortés-Suárez ${ }^{* * * *}$
}

DOl: https://doi.org/10.22458/rb.v32i1.3530

Recibido- Received: 03/11/2020 / Corregido- Revised:01/12/2020 / Aceptado- Accepted: 25/03/2021

\section{RESUMEN}

El artículo recoge la experiencia de participación articulada entre escolares y la comunidad en general en un proceso de siembra y seguimiento de plantas nativas en el área de influencia del Macizo de Iguaque para su recuperación en el municipio de Villa de Leyva, departamento de Boyacá (Colombia). Así como también la elaboración de un instrumento guía, producto del proceso citado, y la socialización del proceso en su totalidad.

Palabras clave: degradación; educación; vegetación; alumnos.

\section{ABSTRACT}

This article presents the experience of articulated participation between school children and the community in general in the process of planting and monitoring native plants in the area of influence of the Iguaque Massif in the municipality of Villa de Leyva, Boyacá department, for its recovery. The paper also presents the elaboration of a guiding instrument, as a result of the whole aforementioned process and its socialization.

Keywords: degradation; education; vegetation; students.

* Estudiante del grado 11 de la Institución Educativa Gimnasio Campestre Santo Domingo, Villa de Leyva, Colombia. lorenareinaf11@gmail.com ID: https://orcid.org/0000-0003-3032-2644

**Estudiante del grado 11 de la Institución Educativa Gimnasio Campestre Santo Domingo, Villa de Leyva, Colombia. andres1cuadrado@gmail.com ID: https://orcid.org/0000-0001-7693-158X

*** Especialista en Gerencia Ambiental y Gestión del Riesgo de Desastres. Presidente, Fundación Ecohumana para la Promoción del Desarrollo Humano Integral Sostenible, Villa de Leyva, Colombia. presidencia@fundacionecohumana.org ID: https://orcid.org/0000-0002-7342-1119

**** Máster en Conservación y Manejo de Vida Silvestre. Investigador Asociado, Fundación Ecohumana para la Promoción del Desarrollo Humano Integral Sostenible, Villa de Leyva, Colombia.sigc@fundacionecohumana.org, javi1885@gmail.com ID: https://orcid.org/0000-0001-6132-6977 


\section{Introducción}

El Macizo de Iguaque se encuentra localizado en el departamento de Boyacá, entre los municipios de Villa de Leyva, Arcabuco, Sachica, Chiquiza y Gachantiva. En una porción de esta cadena montañosa se encuentra ubicada un área protegida, denominada Santuario de Fauna y Flora de Iguaque, la cual forma parte del Sistema de Parques Nacionales de Colombia. El macizo presenta, entre otras características, una representación importante de la vegetación andina de tipo xerofítico del país, y diversos tipos de ecosistemas de paramo, subpáramo y bosques andinos y altoandinos, con un sistema de lagunas característico de estos ecosistemas, que además poseen valor y significado cultural y paisajístico debido a que fue el origen de la civilización Muisca (Ministerio de Medio Ambiente Vivienda y Desarrollo Territorial -MAVDT- y Unidad Administrativa Especial Sistema de Parques Nacionales Naturales -UAESPNN-, 2008; Rubio et al., 2014).

Esta cadena montañosa ha sufrido una degradación ambiental reiterada, ocasionada por diferentes factores como los incendios forestales (antrópicos y naturales), los cuales, según O. Vargas-Ríos (comunicación personal, 15/08/2013) han generado como efecto directo un proceso de sabanización con plantas adaptadas al fuego, que interrumpen el ciclo de regeneración natural y promueven a su vez nuevos incendios, así como procesos de desertificación y desertización (MAVDT, 2005).

Lo anterior, propone un escenario importante para la participación local y en particular para la educación escolarizada, aumentando el conocimiento y profundizando sobre la comprensión del territorio y sus problemáticas de una manera crítica y reflexiva (Beltrán y Giraldo, 2009; Chaparro, 2003; Cerda, 2001; Jurado, 2003; Segura, 2003), a partir de acciones positivas que puedan ayudar a resolver la situación planteada (UNESCO et al., 1997), y que a su vez genere un sentido de apropiación de los elementos que conforman su entorno (Campos y Niño, 2009; Córdoba y Vargas, 2009). Por ello, el objetivo del presente trabajo fue desarrollar un proceso de siembra y seguimiento de plantas nativas en el área de influencia del Macizo de lguaque promoviendo la participación escolar y comunitaria para la elaboración de un instrumento guía que aportara a la recuperación y la conservación del macizo y su área de influencia.

\section{Metodología}

El proyecto se desarrolló entre el mes de abril y septiembre de 2014, como parte de un trabajo para optar al título de bachiller académico en la institución educativa Gimnasio Campestre Santo Domingo (IEGCSD) del municipio de Villa de Leyva, Boyacá, Colombia. La participación articulada entre estudiantes, docentes, directivas, padres de familia y la ONG ambiental Fundación Ecohumana, fue una de las grandes motivaciones del trabajo, el cual se desarrolló a partir de cuatro actividades que se describen a continuación.

\section{Actividades}

Siembra de plantas nativas en el Macizo de Iguaque: Se realizó en un predio privado perteneciente al hotel El Duruelo, en el área de influencia del Macizo de Iguaque, municipio de Villa de Leyva, departamento de Boyacá, Colombia. Para la actividad se involucró a los estudiantes de los grados $6 .^{\circ}, 7 .^{\circ}, 8 .^{\circ}, 9^{\circ}$, $100^{\circ}$ y $11 .^{\circ}$ de la IEGCSD, así como también a 
profesores, profesoras y padres de familia, entre otros actores del territorio.

El sitio de siembra fue seleccionado debido a que presentaba un nivel de humedad del suelo importante para el crecimiento de las plantas, y un estrato vegetal arbóreo (plantación de eucalipto) que podría disminuir el exceso de luz y viento para las plantas sembradas. En esta zona, se localizaron y marcaron las tres parcelas demostrativas donde se sembrarían las 200 plantas nativas seleccionadas, pertenecientes a seis especies donadas por la Fundación Ecohumana: gaque (Clusia multiflora), cajeto (Citharexylum subflavescens) y cucharo blanco (Myrsine guianensis), herrerum (Ficus velutina), camiseto (Baccharis nitida) y sangregado (Croton magdalenensis).

El proceso de siembra inicio desde el transporte de las plantas desde el Vivero Bochica, hasta la zona de siembra. El transporte de las plantas se realizó a través de camioneta hasta la zona más cercana del área de siembra, a partir de lo cual las plantas fueron transportadas por los participantes en canastillas y colocadas en condiciones de sombra mientras eran sembradas.
En el área de siembra, se realizó la respectiva limpieza del lugar donde se colocarían finalmente las plantas, retirando palos, pasto 0 maleza y colocándolos a un lado ya que serían de utilidad posteriormente en el proceso de siembra. Seguido, se efectuó la abertura de los huecos para sembrar las plantas, teniendo en cuenta que la distancia entre los huecos debía ser de entre dos y tres metros (dos o tres pasos largos), así como también que los huecos deben ser dos veces el ancho y el largo de la bolsa de la planta, lo cual permitiría que las raíces se acomodaran fácilmente y pudieran expandirse.

Antes de retirar la bolsa para la siembra de la planta, se tuvo en cuenta que la tierra estuviera húmeda para que pudiera adherirse a las raíces fácilmente. Las bolsas fueron retiradas con las plantas ubicadas en el suelo cerca del hoyo, evitando desperdiciar la tierra contenida, así como también cuidando que las raíces no se rompieran al quitarlas (figura 1). Posteriormente, los huecos donde se ubicaron las plantas fueron tapados y se comprimió la tierra fuertemente con las manos, para así evitar la formación de espacios con aire. Adicionalmente, los pastos, las malezas y los

\section{Figura 1}

Proceso de siembra de cajeto (Citharexylum subflavescens) y cucharo blanco (Myrsine guianensis) por parte de los estudiantes en el área de influencia del Macizo de lguaque
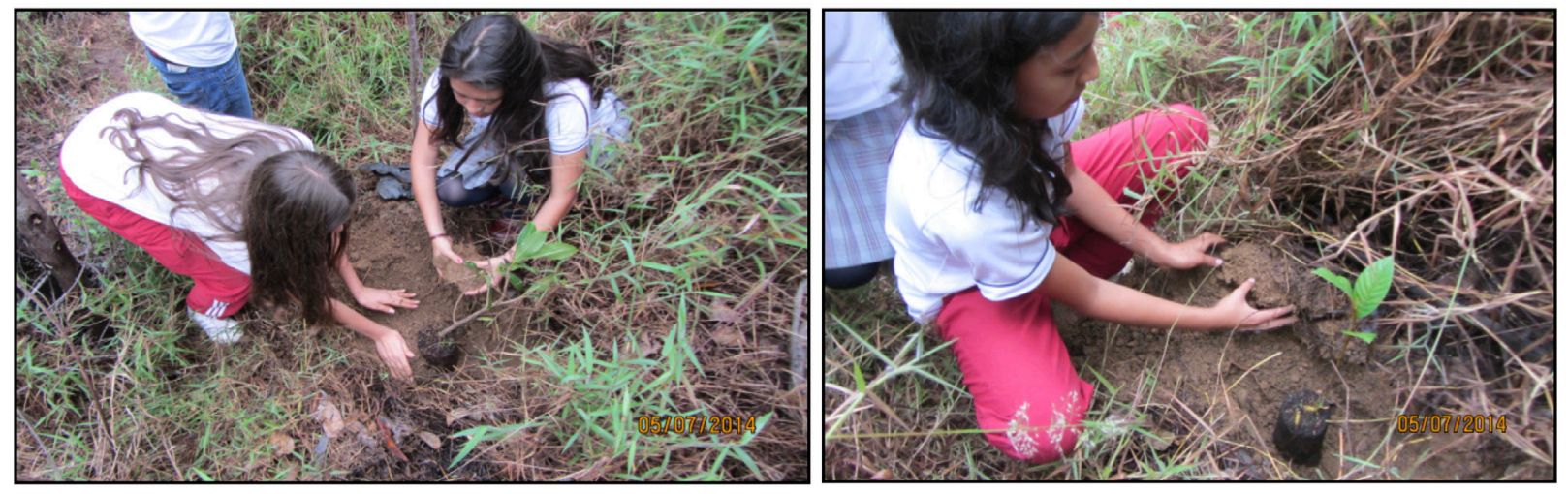

Nota. Fotografías tomadas por Juan Carlos Salas (julio, 2014). 
palos retirados para la limpieza de la zona de siembra en pasos anteriores, fueron utilizados para cubrir la tierra con el fin de evitar que los rayos del sol llegaran directamente a la tierra y secaran la planta.

Etiquetado de plantas y seguimiento post siembra: Se desarrolló esta actividad para las tres parcelas demostrativas. El seguimiento post-siembra se realizó a través de observaciones donde se revisó el estado fitosanitario de las plantas en las tres parcelas, y se registraron datos básicos adicionales sobre su edad de siembra, altura y número de hojas (figura 2). Las actividades solo involucraron a los autores del presente trabajo.

Elaboración de material impreso: Como producto de la actividad de siembra y seguimiento de las plantas nativas se elaboró una

Figura 2

Seguimiento post-siembra de las parcelas demostrativas en el área de influencia del Macizo de lguaque
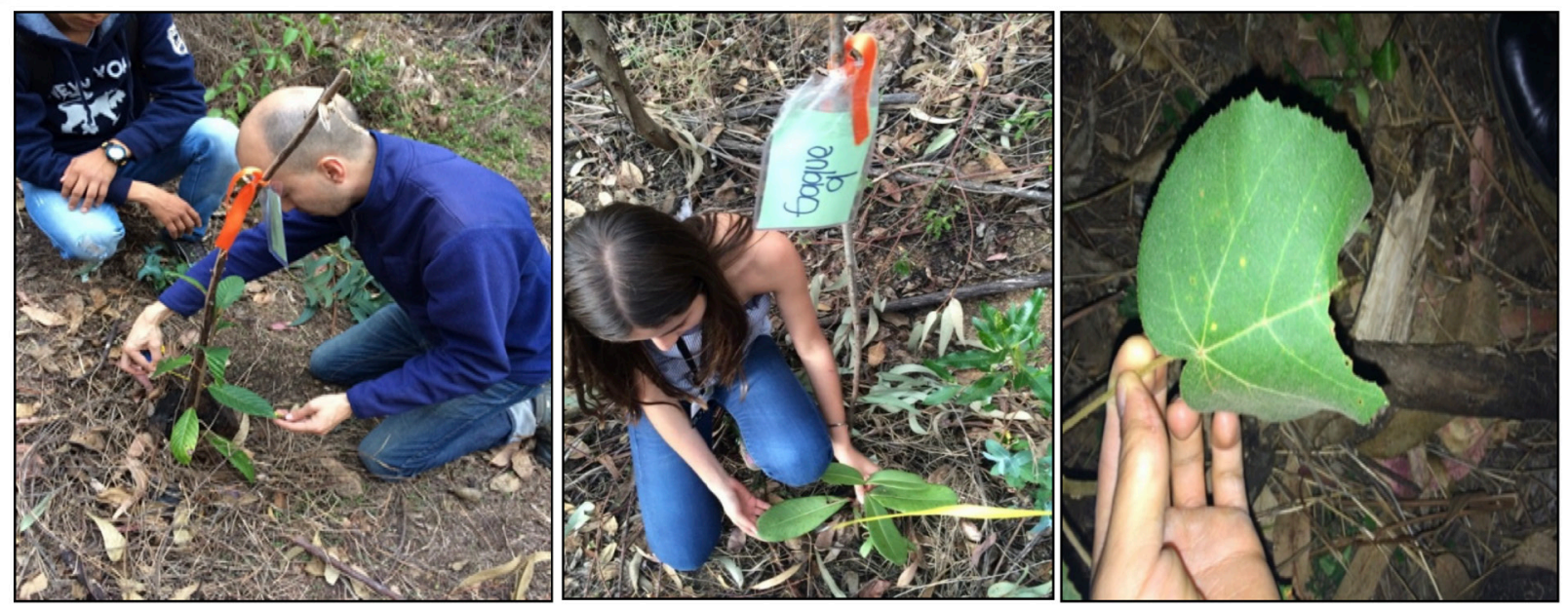

Nota. Fotografías tomadas por Andrés Felipe Cuadrado (agosto, 2014).

cartilla ecológica, con el propósito de contribuir, a través de un instrumento, con los procesos de recuperación ambiental de zonas degradadas en el área de influencia del Macizo de Iguaque en Villa de Leyva, Boyacá (figura 3).

Socialización del proceso: Se realizó la socialización del proceso de siembra de las plantas nativas y de la cartilla ecológica, de la cual se repartieron 30 ejemplares a estudiantes de primaria, secundaria, cuerpo docente y rector de la Escuela Luis Carlos Galán, Colegio Sabana e IEGCSD del municipio de Villa de Leyva, departamento de Boyacá. Cabe mencionar que como parte de una iniciativa escolar también se socializo el proyecto a través de la radio (figura 4).

\section{Evaluación de la experiencia}

Para la fase de siembra, de 64 estudiantes de secundaria de la IEGCSD, 13 participaron activamente del proceso de siembra de las plantas nativas, además de padres de familia y docentes de la institución educativa, entre otros. Es importante resaltar que el acercamiento de los diferentes actores a la actividad de recuperación resultó ser un proceso innovador y estimulante; ya que la gran mayoría 


\section{Figura 3}

Cartilla ecológica para desarrollar actividades de siembra en zonas degradadas del área de influencia en el Macizo de Iguaque
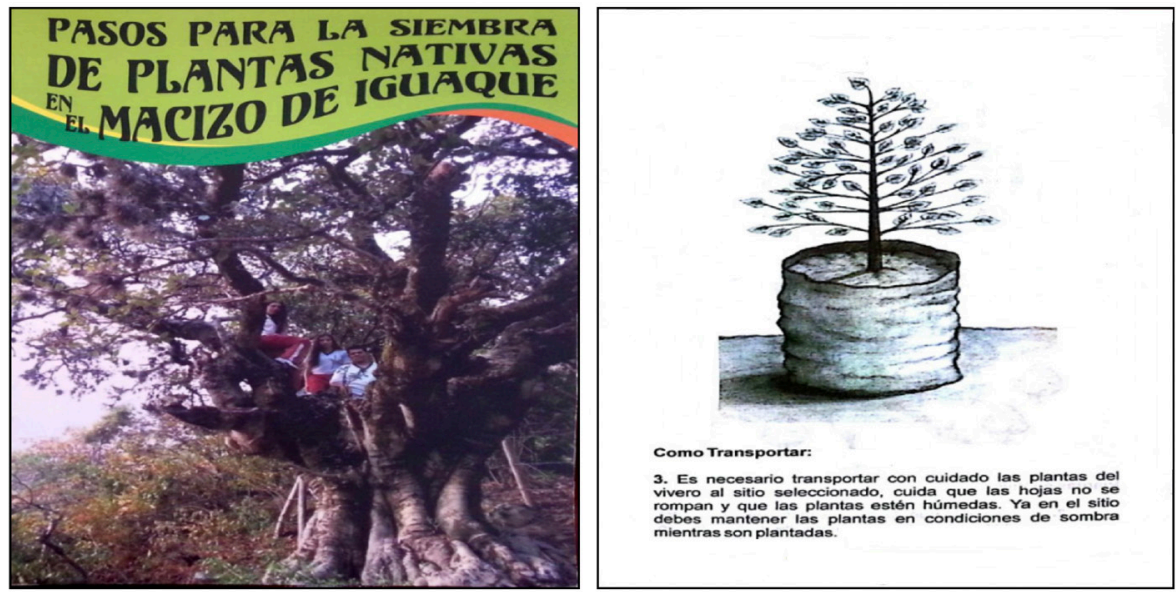

Nota. Fotografías tomadas por Javier E. Cortés (noviembre, 2014).

Figura 4

Socialización del proceso de siembra y seguimiento de plantas nativas en el Macizo de lguaque
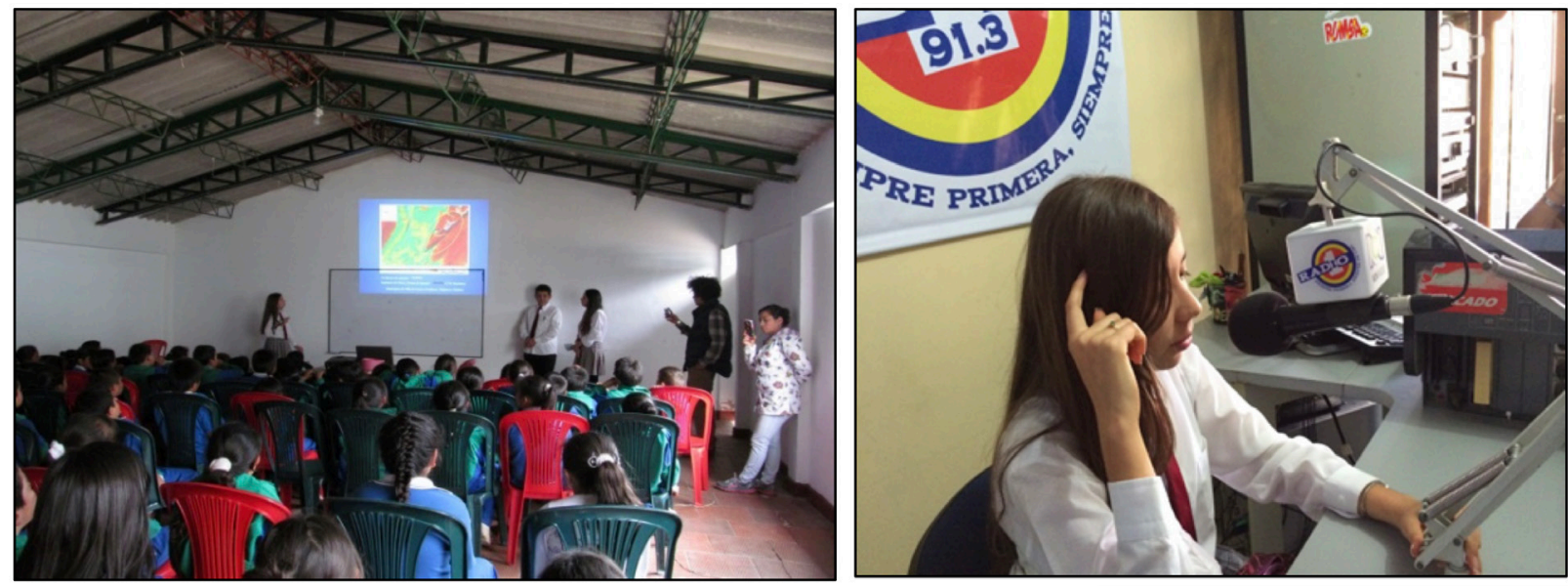

Nota. Fotografías tomadas por Juan Carlos Salas (setiembre, 2014).

nunca había sembrado una planta en su vida y tampoco conocía de los múltiples beneficios que esta actividad podría brindarles en términos del mejoramiento de su calidad de vida. Cabe destacar que la actividad tuvo un valor agregado importante, ya que sirvió como soporte metodológico de las actividades de siembra desarrolladas por parte del Comité Ambiental Dominico de la IEGCSD.
La fase de seguimiento no fue altamente participativa, sin embargo, a través del proceso se aprendió a registrar datos básicos para el seguimiento de las plantas considerando aspectos como su estado fitosanitario, entre otros. Dentro de los datos registrados se encontró que la gran mayoría de plantas presentaban un buen estado fitosanitario; no obstante, varias fueron reportadas con 
hojas de color amarillo, naranja y café debido a la deficiencia de nutrientes en el suelo, así como también a problemas de herbívora en las hojas. Lo anterior, pudo deberse, además de las causas expuestas, a la falta de mantenimiento de las plantas en las parcelas demostrativas.

La elaboración del material impreso (cartilla ecológica) sirvió como actividad de retroalimentación del proceso y afianzamiento de nuevo conocimiento de todos los que participamos del mismo, así como de material de consulta para los alumnos e instrumento pedagógico para los docentes. Por último, la actividad de socialización del proceso incentivó la participación positiva de las diferentes instituciones educativas $y$, en particular, de los docentes y alumnos a través de preguntas y discusiones en un entorno extracurricular.

Cabe resaltar la cobertura de la iniciativa desarrollada por parte de medios de comunicación como la radio, la cual encontró inspiradora la propuesta, ya que a través de ella los jóvenes le comunicaban un mensaje a la comunidad de compromiso y responsabilidad con la recuperación ambiental de su entorno, factor vital de la sostenibilidad del territorio.

\section{Reflexiones finales}

Aunque el ejercicio cumplió con sus propósitos iniciales, también permitió observar aspectos que en un futuro podrían contribuir a un impacto positivo mayor. En este sentido, es importante establecer una fase de evaluación del conocimiento ambiental de los estudiantes o participantes, tanto al iniciar como al finalizar el proceso, lo cual facilita realizar un análisis situacional más profundo.

Por otro lado, hubiese sido una acción más representativa para los participantes el iniciar con otro tipo de estrategias como la germinación y el desarrollo en vivero, para luego pasar a la siembra. Esto generaría no solo una mayor apropiación del proceso por parte de los participantes, sino también de la realidad ambiental y de los elementos que conforman su entorno.

Aunque la fase de seguimiento se desarrolló de una manera básica en cuanto a la toma de datos, es indispensable, como parte del proceso participativo, involucrar a un mayor número de personas que puedan desarrollar acciones de mantenimiento en las plantas sembradas; lo cual garantiza la continuidad y consolidación del proceso a largo plazo, a través del conocimiento que poseen las poblaciones humanas locales.

Igualmente, es necesaria la vinculación de la administración pública en este tipo de proyectos educativos, pues permite la institucionalización del proceso en otras entidades educativas del municipio. 


\section{Referencias}

Beltrán, H. y Giraldo, G. (2009). Elaboración de Proyectos de Aula. En: Talleres deformación: Expediciones Botánicas siglo XXI aprendiendo Ciencias con José Celestino Mutis editado por Beltrán, H. L., Campos, C., Castaño, S., Córdoba, G. y Giraldo, L. A, 20-24. Bogotá, D.C: Ministerio de Educación Nacional (MEN), Universidad Distrital Francisco José de Caldas.

Campos, L. y Niño, G. (2009). Elaboración de herbarios virtuales. En: Talleres de formación: Expediciones Botánicas siglo XXI aprendiendo Ciencias con José Celestino Mutis editado por Beltrán, H. L., Campos, C., Castaño, S., Córdoba, G. y Giraldo, L. A, 20-24. Bogotá, D.C: Ministerio de Educación Nacional (MEN), Universidad Distrital Francisco José de Caldas.

Cerda, H. (2001). El Proyecto de aula: El Aula como un Sistema de Investigación y Construcción de Conocimientos. Bogotá. D.C: MAGISTERIO.

Chaparro, C. (2003). Proyectos de Aula vs. Homogenización. Revista Internacional Magisterio, (2): 34-36.

Córdoba, S. y Vargas, M. (2009). Reverdecimiento Escolar. En: Talleres de formación: Expediciones Botánicas siglo XXI aprendiendo Ciencias con José Celestino Mutis editado por Beltrán, H. L., Campos, C., Castaño, S., Córdoba, G. y Giraldo,L. A, 34-42. Bogotá, D.C: Ministerio de Educación Nacional (MEN), Universidad Distrital Francisco José de Caldas.
Jurado, F. (2003). La educación por proyectos: una pedagogía para la conjetura. Revista Internacional Magisterio, (2): 18-22.

Ministerio de Ambiente, Vivienda y Desarrollo Territorial (MAVDT), The Global Mechanism, UNCCD y PNUD. (2005). Plan De Acción Nacional de Lucha Contra La Desertificación y la Sequía en Colombia. Bogotá: Dirección de Ecosistemas-MAVDT.

Ministerio de Medio Ambiente Vivienda y Desarrollo Territorial (MAVDT) y Unidad Administrativa Especial Sistema de Parques Nacionales Naturales (UAESPNN). (2006). Plan de manejo del Santuario de Flora y Fauna lguaque. Bogotá D. C: MAVDT.

Rubio, T. F., Cárdenas, F. y Pulgarín, Y. (2014). Santuario de Fauna y Flora de Iguaque. http://herenciamia.org/ ricaurte/items/show/11

Segura, D. (2003). Los proyectos de aula más allá de una estrategia didáctica. Revista Intencional Magisterio, (2): 31-33.

UNESCO, PNUMA e ICCE. (1997). Actividades de Educación Ambiental para las Escuelas Primarias. Sugerencias para la confección y empleo de material de bajo costo. En: Programa Internacional de Educación Ambiental editado por UNESCO-PNUMA, 4-99. Santiago de Chile: Centro Internacional de Educación para la Conservación para el Programa Internacional de Educación Ambiental (PIEA). 